\title{
INCREMENTAL LEAST SQUARES METHODS AND THE EXTENDED KALMAN FILTER*
}

\author{
DIMITRI P. BERTSEKAS ${ }^{\dagger}$
}

\begin{abstract}
In this paper we propose and analyze nonlinear least squares methods which process the data incrementally, one data block at a time. Such methods are well suited for large data sets and real time operation and have received much attention in the context of neural network training problems. We focus on the extended Kalman filter, which may be viewed as an incremental version of the Gauss-Newton method. We provide a nonstochastic analysis of its convergence properties, and we discuss variants aimed at accelerating its convergence.
\end{abstract}

Key words. optimization, least squares, Kalman filter

AMS subject classifications. $93-11,90 \mathrm{C} 30,65 \mathrm{~K} 10$

1. Introduction. We consider least squares problems of the form

$$
\begin{aligned}
& \operatorname{minimize} \quad f(x)=\|g(x)\|^{2}=\sum_{i=1}^{m}\left\|g_{i}(x)\right\|^{2} \\
& \text { subject to } \quad x \in \Re^{n},
\end{aligned}
$$

where $g$ is a continuously differentiable function with component functions $g_{1}, \ldots, g_{m}$ s where $g_{i}: \Re^{n} \rightarrow \Re^{r_{i}}$. Here we write $\|z\|$ for the usual Euclidean norm of a vector $z$, that is, $\|z\|=\sqrt{z^{\prime} z}$, where the prime denotes transposition. We also write $\nabla g_{i}$ for the $n \times r_{i}$ gradient matrix of $g_{i}$ and $\nabla g$ for the $n \times\left(r_{1}+\cdots+r_{m}\right)$ gradient matrix of $g$. Least squares problems very often arise in contexts where the functions $g_{i}$ correspond to measurements that we are trying to fit with a model parameterized by $x$. Motivated by this context, we refer to each component $g_{i}$ as a data block, and we refer to the entire function $g=\left(g_{1}, \ldots, g_{m}\right)$ as the data set.

One of the most common iterative methods for solving least squares problems is the Gauss-Newton method, given by

$$
x^{k+1}=x^{k}-\alpha^{k}\left(\nabla g\left(x^{k}\right) \nabla g\left(x^{k}\right)^{\prime}\right)^{-1} \nabla g\left(x^{k}\right) g\left(x^{k}\right)
$$

where $\alpha^{k}$ is a positive stepsize, and we assume that the $n \times n$ matrix $\nabla g\left(x^{k}\right) \nabla g\left(x^{k}\right)^{\prime}$ is invertible. The case $\alpha^{k}=1$ corresponds to the pure form of the method, where $x^{k+1}$ is obtained by linearizing $g$ at the current iterate $x^{k}$ and minimizing the norm of the linearized function, that is,

$$
x^{k+1}=\arg \min _{x \in \Re^{n}}\left\|g\left(x^{k}\right)+\nabla g\left(x^{k}\right)^{\prime}\left(x-x^{k}\right)\right\|^{2} \quad \text { if } \alpha^{k}=1 .
$$

In problems where there are many data blocks, the Gauss-Newton method may be ineffective because the size of the data set makes each iteration very costly. For such problems it may be much better to use an incremental method that does not

*Received by the editors May 27, 1994; accepted for publication (in revised form) April 4, 1995. This research was supported by NSF grant 9300494-DMI.

†Department of Electrical Engineering and Computer Science, Massachusetts Institute of Technology, Cambridge, MA 02139 (dimitrib@mit.edu). 
wait to process the entire data set before updating $x$, as discussed in [Ber95]. Instead, the method cycles through the data blocks in sequence and updates the estimate of $x$ after each data block is processed. A further advantage is that estimates of $x$ become available as data is accumulated, making the approach suitable for real time operation. Such methods include the Widrow-Hoff least-mean-square (LMS) algorithm [WiH60], [WiS85] for the case where the data blocks are linear, and other steepest-descent-like methods for nonlinear data blocks that have been used extensively for the training of neural networks under the generic name of backpropagation methods. A cycle through the data set of a typical example of such a method starts with a vector $x^{k}$ and generates $x^{k+1}$ according to

$$
x^{k+1}=\psi_{m},
$$

where $\psi_{m}$ is obtained at the last step of the recursion

$$
\psi_{i}=\psi_{i-1}-\alpha^{k} \nabla g_{i}\left(\psi_{i-1}\right) g_{i}\left(\psi_{i-1}\right), \quad i=1, \ldots, m,
$$

$\alpha^{k}$ is a positive stepsize, and $\psi_{0}=x^{k}$.

Backpropagation methods are often effective, and they are supported by stochastic [PoT73], [Lju77], [KuC78], [Pol87], [BeT89], [Whi89a], [Whi89b], [Gai93], [BeT96] as well as deterministic convergence analyses [Luo91], [Gri93], [LuT93], [MaS94], [Man93], [BeT96]. The main difference between stochastic and deterministic methods of analysis is that the former apply to an infinite data set (one with an infinite number of data blocks) satisfying some statistical assumptions, while the latter apply to a finite data set. There are also parallel asynchronous versions of backpropagation methods and corresponding stochastic [Tsi84], [TBA86], [BeT89], [Gai93] as well as deterministic convergence results [Tsi84], [TBA86], [BeT89], [MaS94]. However, backpropagation methods typically have a slow convergence rate not only because they are first-order steepest-descent-like methods, but also because they require a diminishing stepsize $\alpha^{k}=O(1 / k)$ for convergence. If $\alpha^{k}$ is instead taken to be a small constant, an oscillation within each data cycle typically arises, as shown by [Luo91].

In this paper we focus on methods that combine the advantages of backpropagation methods for large data sets with the often superior convergence rate of the Gauss-Newton method. We thus consider an incremental version of the Gauss-Newton method, which operates in cycles through the data blocks. The $(k+1)$ st cycle starts with a vector $x^{k}$ and a positive semidefinite matrix $H^{k}$ to be defined later, then updates $x$ via a Gauss-Newton-like iteration aimed at minimizing

$$
\lambda\left(x-x^{k}\right)^{\prime} H^{k}\left(x-x^{k}\right)+\left\|g_{1}(x)\right\|^{2}
$$

where $\lambda$ is a scalar with

$$
0<\lambda \leq 1
$$

then updates $x$ via a Gauss-Newton-like iteration aimed at minimizing

$$
\lambda^{2}\left(x-x^{k}\right)^{\prime} H^{k}\left(x-x^{k}\right)+\lambda\left\|g_{1}(x)\right\|^{2}+\left\|g_{2}(x)\right\|^{2},
$$

and similarly continues, with the $i$ th step consisting of a Gauss-Newton-like iteration aimed at minimizing the weighted partial sum

$$
\lambda^{i}\left(x-x^{k}\right)^{\prime} H^{k}\left(x-x^{k}\right)+\sum_{j=1}^{i} \lambda^{i-j}\left\|g_{j}(x)\right\|^{2} .
$$


In particular, given $x^{k}$, the $(k+1)$ st cycle sequentially generates the vectors (4)

$\psi_{i}=\arg \min _{x \in \Re^{n}}\left\{\lambda^{i}\left(x-x^{k}\right)^{\prime} H^{k}\left(x-x^{k}\right)+\sum_{j=1}^{i} \lambda^{i-j}\left\|\tilde{g}_{j}\left(x, \psi_{j-1}\right)\right\|^{2}\right\}, \quad i=1, \ldots, m$,

and sets

$$
x^{k+1}=\psi_{m}
$$

where $\tilde{g}_{j}\left(x, \psi_{j-1}\right)$ are the linearized functions

$$
\tilde{g}_{j}\left(x, \psi_{j-1}\right)=g_{j}\left(\psi_{j-1}\right)+\nabla g_{j}\left(\psi_{j-1}\right)^{\prime}\left(x-\psi_{j-1}\right)
$$

and $\psi_{0}$ is the estimate of $x$ at the end of the $k$ th cycle:

$$
x^{k}=\psi_{0}
$$

As will be seen later, the quadratic minimizations above can be efficiently implemented using the recursive Kalman filter formulas.

The most common version of the preceding algorithm is obtained when the matrices $H^{k}$ are updated by the recursion

$$
H^{k+1}=\lambda^{m} H^{k}+\sum_{j=1}^{m} \lambda^{m-j} \nabla g_{j}\left(\psi_{j-1}\right) \nabla g_{j}\left(\psi_{j-1}\right)^{\prime}
$$

Then for $\lambda=1$ and $H^{0}=0$, the method becomes the well-known extended Kalman filter (EKF for short) specialized to the case where the state of the underlying dynamical system stays constant and the measurement equation is nonlinear. The EKF was originally conceived as a method for estimating parameters from nonlinear measurements that are generated in real time. The basic idea of the method is to linearize each new measurement around the current value of the estimate and treat the measurement as if it were linear (cf. eq. (4)). The estimate is then corrected to account for the new (linearized) measurement using the convenient Kalman filter formulas (see Lemma 1). The algorithm considered here cycles repeatedly through the data set and is sometimes called the iterated extended Kalman filter. For the problem of estimating the state of a dynamic system, a cycle through the data set involves solving a problem of smoothing the estimate of the state trajectory before starting a new cycle (see, e.g., [Bel94]). The matrix $H^{k}$ has the meaning of the inverse of an approximate error covariance of the estimate $x^{k}$. In the case $\lambda<1$, the effect of old data blocks is discounted, and successive estimates produced by the method tend to change more rapidly. In this way one may obtain a faster rate of progress of the method, and this is the main motivation for considering $\lambda<1$.

The EKF has been used extensively in a variety of control and estimation applications (see, e.g., [AWT69], [Jaz70], [Meh71], [THS77], [AnM79], [WeM80]) and has also been suggested for the training of neural networks (see, e.g., [WaT90] and [RRK92]). The version of the algorithm (4)-(8) with $\lambda<1$ has also been proposed by Davidon [Dav76]. Unaware of the earlier work in the control and estimation literature, Davidon described the qualitative behavior of the method together with favorable computational experience for problems with large data sets, but gave no convergence 
analysis. The first convergence analysis of the EKF was given by Ljung [Lju79], who assuming $\lambda=1$ used a stochastic formulation (i.e., an infinite data set) and the ODE approach of [Lju77] to prove satisfactory convergence properties for a version of the EKF that is closely related to the one considered here (Theorem 6.1 of [Lju79], which assumes a stationary measurement equation and additive noise). Ljung also showed that the EKF, when applied to more complex models where the underlying dynamic system is linear but its dynamics depend on $x$, exhibits complex behavior, including the possible convergence to biased estimates. For such models he suggested the use of a different formulation of the least squares problem involving the innovations process (see also [Urs80]). The algorithms and analysis of the present paper apply to any type of deterministic least squares problem, and thus also apply to Ljung's innovations-based formulation.

A deterministic analysis of the EKF method (4)-(8), where $\lambda<1$, was given in Pappas's Master's thesis [Pap82]. He considered only the special case where $\min _{x}\|g(x)\|=0$ and showed that the EKF converges locally to a nonsingular solution of the system $g(x)=0$ at a rate that is linear with convergence ratio $\lambda^{m}$. He also argued by example that when $\lambda<1$ and $\min _{x}\|g(x)\|>0$, the iterates $\psi_{i}$ produced by the EKF within each cycle generally oscillate with a "size" of oscillation that diminishes as $\lambda$ approaches 1 .

The purpose of this paper is to provide a deterministic analysis of the convergence properties of the EKF for the general case where $\min _{x}\|g(x)\|$ is not necessarily zero. Our analysis is complicated by the lack of an explicit stepsize in the algorithm. In the case where $\lambda=1$ we show that the limit points of the generated sequence $\left\{x^{k}\right\}$ by the $\mathrm{EKF}$ are stationary points of the least squares problem. The idea of the proof is to show that the method involves an implicit stepsize of order $O(1 / k)$ and then to apply arguments similar to those used by Tsitsiklis [Tsi84] and Tsitsiklis, Bertsekas, and Athans [TBA86] in their analyses of asynchronous distributed gradient methods, and by Mangasarian and Solodov [MaS94] in their convergence proof of an asynchronous parallel backpropagation method. To improve the rate of convergence of the method, which is sublinear and typically slow, we suggest a convergent and empirically faster variant where $\lambda$ is initially less than 1 and is progressively increased toward 1 .

In addition to dealing more naturally with the case of a finite data set, a nice aspect of the deterministic analysis is that it decouples the stochastic modeling of the data generation process from the algorithmic solution of the least squares problem. In other words, the EKF discussed here will (typically) find a least squares solution even if the least squares formulation is inappropriate for the corresponding real parameter estimation problem. This is a valuable insight because it is sometimes thought that convergence of the EKF depends on the validity of the underlying stochastic model assumptions.

2. The EKF. When the data blocks are linear functions, it takes a single pure Gauss-Newton iteration to find the least squares estimate. This iteration can be implemented as an incremental algorithm, the Kalman filter, which we now describe. Assume that the functions $g_{i}$ are linear and of the form

$$
g_{i}(x)=z_{i}-C_{i} x
$$

where $z_{i} \in \Re^{r_{i}}$ are given vectors and $C_{i}$ are given $r_{i} \times n$ matrices. Let us consider the incremental method that generates the vectors

$$
\psi_{i}=\arg \min _{x \in \Re^{n}} \sum_{j=1}^{i} \lambda^{i-j}\left\|z_{j}-C_{j} x\right\|^{2}, \quad i=1, \ldots, m .
$$


Then the method can be recursively implemented, as shown by the following wellknown proposition (see, e.g., [AnM79]).

Proposition 1 (Kalman filter). Assuming that the matrix $C_{1}^{\prime} C_{1}$ is positive definite, the least squares estimates

$$
\psi_{i}=\arg \min _{x \in \Re^{n}} \sum_{j=1}^{i} \lambda^{i-j}\left\|z_{j}-C_{j} x\right\|^{2}, \quad i=1, \ldots, m,
$$

can be generated by the algorithm

$$
\psi_{i}=\psi_{i-1}+H_{i}^{-1} C_{i}^{\prime}\left(z_{i}-C_{i} \psi_{i-1}\right), \quad i=1, \ldots, m,
$$

where $\psi_{0}$ is an arbitrary vector, and the positive-definite matrices $H_{i}$ are generated by

$$
H_{i}=\lambda H_{i-1}+C_{i}^{\prime} C_{i}, \quad i=1, \ldots, m,
$$

with

$$
H_{0}=0 \text {. }
$$

More generally, for all $\bar{i}<i$ we have

$$
\psi_{i}=\psi_{\bar{i}}+H_{i}^{-1} \sum_{j=\bar{i}+1}^{i} \lambda^{i-j} C_{j}^{\prime}\left(z_{j}-C_{j} \psi_{\bar{i}}\right), \quad i=1, \ldots, m .
$$

The proof of Proposition 1 is obtained by using the following lemma involving two data blocks, the straightforward proof of which is omitted.

LEMMA 1. Let $\zeta_{1}, \zeta_{2}$ be given vectors and $\Gamma_{1}, \Gamma_{2}$ be given matrices such that $\Gamma_{1}^{\prime} \Gamma_{1}$ is positive definite. Then the vectors

$$
\psi_{1}=\arg \min _{x \in \Re^{n}}\left\|\zeta_{1}-\Gamma_{1} x\right\|^{2}
$$

and

$$
\psi_{2}=\arg \min _{x \in \Re^{n}}\left\{\left\|\zeta_{1}-\Gamma_{1} x\right\|^{2}+\left\|\zeta_{2}-\Gamma_{2} x\right\|^{2}\right\}
$$

are also given by

$$
\psi_{1}=\psi_{0}+\left(\Gamma_{1}^{\prime} \Gamma_{1}\right)^{-1} \Gamma_{1}^{\prime}\left(\zeta_{1}-\Gamma_{1} \psi_{0}\right)
$$

and

$$
\psi_{2}=\psi_{1}+\left(\Gamma_{1}^{\prime} \Gamma_{1}+\Gamma_{2}^{\prime} \Gamma_{2}\right)^{-1} \Gamma_{2}^{\prime}\left(\zeta_{2}-\Gamma_{2} \psi_{1}\right),
$$

where $\psi_{0}$ is an arbitrary vector.

The proof of eqs. (12) and (13) of Proposition 1 follows by applying Lemma 1 with the correspondences $\psi_{0} \sim \psi_{0}, \psi_{1} \sim \psi_{\bar{i}}, \psi_{2} \sim \psi_{i}$, and

$$
\zeta_{1} \sim\left(\begin{array}{c}
\sqrt{\lambda^{i-1}} z_{1} \\
\vdots \\
\sqrt{\lambda^{i-\bar{i}}} z_{\bar{i}}
\end{array}\right), \quad \Gamma_{1} \sim\left(\begin{array}{c}
\sqrt{\lambda^{i-1}} C_{1} \\
\vdots \\
\sqrt{\lambda^{i-\bar{i}}} C_{\bar{i}}
\end{array}\right),
$$




$$
\zeta_{2} \sim\left(\begin{array}{c}
\sqrt{\lambda^{i-\bar{i}-1}} z_{\bar{i}+1} \\
\vdots \\
z_{i}
\end{array}\right), \quad \Gamma_{2} \sim\left(\begin{array}{c}
\sqrt{\lambda^{i-\bar{i}-1}} C_{\bar{i}+1} \\
\vdots \\
C_{i}
\end{array}\right),
$$

and by carrying out the straightforward algebra.

Note that the positive definiteness assumption on $C_{1}^{\prime} C_{1}$ in Proposition 1 is needed to guarantee that the first matrix $H_{1}$ is positive definite and hence invertible; then the positive definiteness of the subsequent matrices $H_{2}, \ldots, H_{m}$ follows from eq. (12). As a practical matter, it is possible to guarantee the positive definiteness of $C_{1}^{\prime} C_{1}$ by lumping a sufficient number of measurements into the first data block $\left(C_{1}\right.$ should contain $n$ linearly independent columns). An alternative is to redefine $\psi_{i}$ as

$$
\psi_{i}=\arg \min _{x \in \Re^{n}}\left\{\delta \lambda^{i}\left\|x-\psi_{0}\right\|^{2}+\sum_{j=1}^{i} \lambda^{i-j}\left\|z_{j}-C_{j} x\right\|^{2}\right\}, \quad i=1, \ldots, m,
$$

where $\delta$ is a small positive scalar. Then it can be seen that $\psi_{i}$ is generated by the same equations (11) and (12), except that the initial condition $H_{0}=0$ is replaced by

$$
H_{0}=\delta I,
$$

so that $H_{1}=\delta I+C_{1}^{\prime} C_{1}$ is positive definite even if $C_{1}^{\prime} C_{1}$ is not. Note, however, that in this case the last estimate $\psi_{m}$ is only approximately equal to the least squares estimate $x^{*}$, even if $\lambda=1$ (the approximation error depends on the size of $\delta$ ).

Now consider the general case where the data blocks $g_{i}$ are nonlinear. Then the EKF can be used, and its first cycle can be implemented by means of the Kalman filter equations of Proposition 1. Using formulas (11) and (12) with the identifications

$$
z_{i}=g_{i}\left(\psi_{i-1}\right)-\nabla g_{i}\left(\psi_{i-1}\right)^{\prime} \psi_{i-1}, \quad C_{i}=-\nabla g_{i}\left(\psi_{i-1}\right)^{\prime}
$$

the $k$ th cycle of the EKF can be written in the incremental form

$$
\psi_{i}=\psi_{i-1}-H_{i}^{-1} \nabla g_{i}\left(\psi_{i-1}\right) g_{i}\left(\psi_{i-1}\right), \quad i=1, \ldots, m,
$$

where the matrices $H_{i}$ are generated by

$$
H_{i}=\lambda H_{i-1}+\nabla g_{i}\left(\psi_{i-1}\right) \nabla g_{i}\left(\psi_{i-1}\right)^{\prime}, \quad i=1, \ldots, m,
$$

with

$$
H_{0}=0 \text {. }
$$

To contrast the EKF with the pure form of the Gauss-Newton method (unit stepsize), note that a single iteration of the latter can be written as

$$
x^{k+1}=\arg \min _{x \in \Re^{n}} \sum_{i=1}^{m}\left\|\tilde{g}_{i}\left(x, x^{k}\right)\right\|^{2} .
$$

Using the formulas of Proposition 1 with the identifications

$$
z_{i}=g_{i}\left(x^{k}\right)-\nabla g_{i}\left(x^{k}\right)^{\prime} x^{k}, \quad C_{i}=-\nabla g_{i}\left(x^{k}\right)^{\prime},
$$


we can generate $x^{k+1}$ by an incremental algorithm as

$$
x^{k+1}=\bar{\psi}_{m}
$$

where

$$
\bar{\psi}_{i}=\bar{\psi}_{i-1}-\bar{H}_{i}^{-1} \nabla g_{i}\left(x^{k}\right)\left(g_{i}\left(x^{k}\right)+\nabla g_{i}\left(x^{k}\right)^{\prime}\left(\bar{\psi}_{i-1}-x^{k}\right)\right), \quad i=1, \ldots, m,
$$

$\bar{\psi}_{0}=x^{k}$, and the matrices $\bar{H}_{i}$ are generated by

$$
\bar{H}_{i}=\bar{H}_{i-1}+\nabla g_{i}\left(x^{k}\right) \nabla g_{i}\left(x^{k}\right)^{\prime}, \quad i=1, \ldots, m
$$

with

$$
\bar{H}_{0}=0 \text {. }
$$

Thus, by comparing eqs. (21)-(23) with eqs. (25)-(27), we see that, if $\lambda=1$, a cycle of the EKF through the data set differs from a pure Gauss-Newton iteration only in that the linearization of the data blocks $g_{i}$ is done at the corresponding current estimates $\psi_{i-1}$ rather than at the estimate $x^{k}$ available at the start of the cycle. If the data blocks are linear, the EKF with $\lambda=1$ and the Gauss-Newton iteration coincide and solve the problem in a single cycle through the data set.

3. Convergence of the EKF. We have considered so far a single cycle of the EKF. To obtain an algorithm that cycles through the data set multiple times, there are two basic approaches. The first approach is to reset the matrix $H$ to some fixed matrix $H_{0}$ at the start of each cycle. Unfortunately, the convergence properties of the resulting algorithm are questionable, and one can construct examples where the method diverges, basically because the increments $\psi_{i}-\psi_{i-1}$ produced by the method (cf. eq. (21)) may be too large. One may attempt to correct this behavior by selecting $H_{0}$ to be a sufficiently large multiple of the identity matrix, but this leads to large asymptotic convergence errors (biased estimates), as can be seen through simple examples where the data blocks are linear.

The second approach, which is followed in this paper, is to create a larger data set by concatenating multiple copies of the original data set, that is, by forming what we refer to as the extended data set

$$
\left(g_{1}, g_{2}, \ldots, g_{m}, g_{1}, g_{2}, \ldots, g_{m}, g_{1}, g_{2}, \ldots\right)
$$

The EKF is then applied to the extended data set and takes the form given in the introduction (eqs. (4)-(8)). The algorithm has the form

$$
\begin{gathered}
H_{k m+i}=\lambda H_{k m+i-1}+\nabla g_{i}\left(\psi_{k m+i-1}\right) \nabla g_{i}\left(\psi_{k m+i-1}\right)^{\prime}, \quad i=1, \ldots, m, \\
\psi_{k m+i}=\psi_{k m+i-1}-H_{k m+i}^{-1} \nabla g_{i}\left(\psi_{k m+i-1}\right) g_{i}\left(\psi_{k m+i-1}\right), \quad i=1, \ldots, m,
\end{gathered}
$$

where $H_{0}=0, \psi_{0}=x^{0}$ is an arbitrary vector, and $k$ indexes the current cycle through the data set. Note that while in the above equations $\lambda$ is written as a constant, we will later consider the possibility of changing $\lambda$ in the course of the algorithm. Also, we assume that the matrix $\nabla g_{1}\left(x^{0}\right) \nabla g_{1}\left(x^{0}\right)^{\prime}$ is invertible, so that $H_{1}^{-1}$ is well defined. However, it can be shown that the convergence result to be given shortly 
also holds when $H_{0}$ is any positive-definite matrix, in which case the invertibility of $\nabla g_{1}\left(x^{0}\right) \nabla g_{1}\left(x^{0}\right)^{\prime}$ is unnecessary.

We will show that when $\lambda=1$, the EKF version just described typically converges to stationary points of the least squares problem. The basic reason is that the EKF asymptotically resembles a gradient method with diminishing stepsize of order $O(1 / k)$. To get a sense of this, assume that the EKF is applied to the extended data set (28) with $\lambda=1$. Let us denote by $x^{k}$.the iterate at the end of the $k$ th cycle through the data set, that is,

$$
x^{k}=\psi_{k m}, \quad k=1,2, \ldots
$$

Then, by using eq. (13) with $i=(k+1) m$ and $\bar{i}=k m$, we obtain

$$
x^{k+1}=x^{k}-H_{(k+1) m}^{-1} \sum_{i=1}^{m} \nabla g_{i}\left(\psi_{k m+i-1}\right) g_{i}\left(\psi_{k m+i-1}\right) \text {. }
$$

Now $H_{(k+1) m}$ grows roughly in proportion to $k+1$ because, by eq. (12), we have

$$
H_{(k+1) m}=\sum_{j=0}^{k} \sum_{i=1}^{m} \nabla g_{i}\left(\psi_{j m+i-1}\right) \nabla g_{i}\left(\psi_{j m+i-1}\right)^{\prime} .
$$

It is therefore reasonable to expect that the method tends to make slow progress when $k$ is large, which means that the vectors $\psi_{k m+i-1}$ in eq. (29) are roughly equal to $x^{k}$. Thus, for large $k$, the sum in the right-hand side of eq. (29) is roughly equal to the gradient $\nabla g\left(x^{k}\right) g\left(x^{k}\right)$, while from eq. (30), $H_{(k+1) m}$ is roughly equal to $(k+$ 1) $\nabla g\left(x^{k}\right) \nabla g\left(x^{k}\right)^{\prime}$, where $g=\left(g_{1}, g_{2}, \ldots, g_{m}\right)$ is the original data set. It follows that for large $k$, the EKF iteration (29) can be written approximately as

$$
x^{k+1} \approx x^{k}-\frac{1}{k+1}\left(\nabla g\left(x^{k}\right) \nabla g\left(x^{k}\right)^{\prime}\right)^{-1} \nabla g\left(x^{k}\right) g\left(x^{k}\right),
$$

that is, as an approximate Gauss-Newton iteration with diminishing stepsize. Thus, based on generic properties of gradient methods with diminishing stepsize (see, e.g., [Pol87]), we can expect convergence to stationary points of the least squares problem and a sublinear convergence rate.

When $\lambda<1$, the matrix $H_{i}^{-1}$ generated by the EKF recursion (22) will typically not diminish to zero, and $\left\{x^{k}\right\}$ may not converge to a stationary point of $\sum_{i=1}^{m} \lambda^{m-i}\left\|g_{i}(x)\right\|^{2}$. Furthermore, as the following example shows, the sequences $\left\{\psi_{k m+i}\right\}$ produced by the EKF using eq. (21) may converge to different limits for different $i$.

Example 1. Consider the case where there are two data blocks, $g_{1}(x)=x-c_{1}$ and $g_{2}(x)=x-c_{2}$, where $c_{1}$ and $c_{2}$ are given scalars. Each cycle of the EKF consists of two steps. At the second step of the $k$ th cycle, we minimize

$$
\sum_{i=1}^{k}\left(\lambda^{2 i-1}\left(x-c_{1}\right)^{2}+\lambda^{2 i-2}\left(x-c_{2}\right)^{2}\right),
$$

which is equal to the following scalar multiple of $\lambda\left(x-c_{1}\right)^{2}+\left(x-c_{2}\right)^{2}$ :

$$
\left(1+\lambda^{2}+\cdots+\lambda^{2 k-2}\right)\left(\lambda\left(x-c_{1}\right)^{2}+\left(x-c_{2}\right)^{2}\right) .
$$


Thus, at the second step, we obtain the minimizer of $\lambda\left(x-c_{1}\right)^{2}+\left(x-c_{2}\right)^{2}$ :

$$
\psi_{2 k}=\frac{\lambda c_{1}+c_{2}}{\lambda+1}
$$

At the first step of the $k$ th cycle we minimize

$$
\left(x-c_{1}\right)^{2}+\lambda \sum_{i=1}^{k-1}\left(\lambda^{2 i-1}\left(x-c_{1}\right)^{2}+\lambda^{2 i-2}\left(x-c_{2}\right)^{2}\right),
$$

which is equal to the following scalar multiple of $\left(x-c_{1}\right)^{2}+\lambda\left(x-c_{2}\right)^{2}$ :

$$
\left(1+\lambda^{2}+\cdots+\lambda^{2 k-4}\right)\left(\left(x-c_{1}\right)^{2}+\lambda\left(x-c_{2}\right)^{2}\right),
$$

plus the diminishing term $\lambda^{2 k-2}\left(x-c_{1}\right)^{2}$. Thus, at the first step, we obtain approximately (for large $k$ ) the minimizer of $\left(x-c_{1}\right)^{2}+\lambda\left(x-c_{2}\right)^{2}$ :

$$
\psi_{2 k-1} \approx \frac{c_{1}+\lambda c_{2}}{1+\lambda}
$$

We see, therefore, that within each cycle there is an oscillation around the minimizer $\left(c_{1}+c_{2}\right) / 2$ of $\left(x-c_{1}\right)^{2}+\left(x-c_{2}\right)^{2}$. The size of the oscillation diminishes as $\lambda$ approaches 1.

The preceding example suggests that each sequence $\left\{\psi_{k m+i}\right\}$, where $i=1, \ldots, m$, may converge to a stationary point of the function

$$
f_{i}(x)=\sum_{j=1}^{m} \lambda^{m-j}\left\|g_{j+i}(x)\right\|^{2}, \quad i=1, \ldots, m
$$

where we use the definition

$$
g_{j}(x)=g_{j \bmod (m)+1}(x) \text { if } j>m .
$$

This is readily shown when the data blocks $g_{i}$ are linear in view of the definition of $\psi_{k m+i}$ as the minimizer of

$$
\sum_{j=1}^{k m+i} \lambda^{k m+i-j}\left\|g_{j}(x)\right\|^{2}
$$

which can also be written as

$$
\sum_{j=1}^{i} \lambda^{k m+i-j}\left\|g_{j}(x)\right\|^{2}+\left(1+\lambda^{2}+\cdots+\lambda^{(k-1) m}\right) f_{i}(x) .
$$

Since the leftmost summation above vanishes as $k \rightarrow \infty, \psi_{k m+i}$ minimizes $f_{i}(x)$ asymptotically. In the case of nonlinear data blocks, a related but more complex analysis of the cyclic convergence behavior described above is possible, but this analysis will not be attempted in this paper.

Generally, for a nonlinear least squares problem, the convergence rate tends to be faster when $\lambda<1$ than when $\lambda=1$, essentially because the implicit stepsize does not diminish to zero as in the case $\lambda=1$. For this reason, a hybrid method that uses a 
different value of $\lambda$ within each cycle may work best in practice. One may start with a relatively small $\lambda$ to attain a fast initial rate of convergence, and then progressively increase $\lambda$ toward 1 to attain high solution accuracy. The following proposition shows convergence for the case where $\lambda$ tends to 1 at a sufficiently fast rate.

Proposition 2. Assume that $\nabla g_{i}(x)$ has full rank for all $x$ and $i=1, \ldots, m$, and that for some $L>0$ we have

$$
\left\|\nabla g_{i}(x) g_{i}(x)-\nabla g_{i}(y) g_{i}(y)\right\| \leq L\|x-y\| \quad \forall x, y \in \Re^{n}, i=1, \ldots, m .
$$

Assume also that there is a constant $c>0$ such that the scalar $\lambda$ used in the updating formula (22) within the $k$ th cycle, call it $\lambda_{k}$, satisfies

$$
0 \leq 1-\lambda_{k}^{m} \leq \frac{c}{k} \quad \forall k=1,2, \ldots
$$

Then if the EKF applied to the extended data set (28) generates a bounded sequence of vectors $\psi_{i}$, the sequence $\left\{f\left(x^{k}\right)\right\}$ converges and each of the limit points of $\left\{x^{k}\right\}$ is a stationary point of the least squares problem.

We develop the proof of Proposition 2 through a series of lemmas, all of which implicitly assume the conditions of Proposition 2.

LEMMA 2. There exist positive scalars $c_{1}$ and $c_{2}$ such that for all $k$, the eigenvalues of the matrices $H_{k m}$ lie within the interval $\left[c_{1} k, c_{2} k\right]$.

Proof. We have, using the update formula (22), that

$$
H_{(k+1) m}=\lambda_{k+1}^{m} H_{k m}+\sum_{i=1}^{m} \lambda_{k+1}^{m-i} \nabla g_{i}\left(\psi_{k m+i-1}\right) \nabla g_{i}\left(\psi_{k m+i-1}\right)^{\prime} .
$$

Let $X$ be a compact set containing all vectors $\psi_{i}$ generated by the algorithm, and let $B$ and $b$ be an upper bound and a lower bound, respectively, for the eigenvalues of $\nabla g_{i}(x) \nabla g_{i}(x)^{\prime}$ as $x$ ranges over $X$. From eq. (34), it is seen by induction that all eigenvalues of $H_{k m}$ are less than or equal to $c_{2} k$ with $c_{2}=m B$. Furthermore, if $v_{k}$ is the smallest eigenvalue of $H_{k m}$, then from eqs. (33) and (34) it is seen by induction that

$$
v_{k+1} \geq\left(1-\frac{c}{k+1}\right) v_{k}+\left(1-\frac{c}{k+1}\right) m b \quad \forall k \geq 1
$$

Using this relation, we will prove that $v_{k} \geq k \beta$ for a sufficiently small but positive value of $\beta$. Indeed, let $\bar{k}$ be the minimal positive integer $k$ such that $c / k<1$, and let $\beta$ be any positive scalar such that

$$
\beta \leq \frac{(\bar{k}+1-c) m b}{\bar{k}+1+\bar{k} c}
$$

From eq. (35), it is seen that if $v_{\bar{k}} \geq \beta \bar{k}$, then

$$
\begin{aligned}
v_{\bar{k}+1} & \geq\left(1-\frac{c}{\bar{k}+1}\right) \beta \bar{k}+\left(1-\frac{c}{\bar{k}+1}\right) m b \\
& =\beta(\bar{k}+1)+\frac{(\bar{k}+1-c) m b}{\bar{k}+1}-\frac{(\bar{k}+1+\bar{k} c) \beta}{\bar{k}+1} \\
& \geq \beta(\bar{k}+1) .
\end{aligned}
$$


Similarly, it is shown that $v_{k} \geq \beta k$ for all $k \geq \bar{k}$. Thus, by taking $\beta$ equal to the scalar $c_{1}$ given below,

$$
c_{1}=\min \left\{\frac{(\bar{k}+1-c) m b}{\bar{k}+1+\bar{k} c}, \min _{k=1, \ldots, \bar{k}} \frac{v_{k}}{k}\right\},
$$

we see that $v_{k} \geq c_{1} k$ for all $k \geq 1$.

We will use the notation

$$
f(x)=\frac{1}{2} \sum_{i=1}^{m}\left\|g_{i}(x)\right\|^{2}
$$

from which we have

$$
\nabla f(x)=\sum_{i=1}^{m} \nabla g_{i}(x) g_{i}(x)
$$

The next lemma shows that the vector that is multiplied by $H_{(k+1) m}^{-1}$ to obtain the direction used by the EKF (cf. eqs. (13) and (29)) differs from the gradient $\nabla f\left(x^{k}\right)$ by a relatively small amount.

LEMMA 3. Let

$$
e^{k}=\nabla f\left(x^{k}\right)-\sum_{i=1}^{m} \lambda_{k+1}^{m-i} \nabla g_{i}\left(\psi_{k m+i-1}\right) g_{i}\left(\psi_{k m+i-1}\right) .
$$

Then there exists a scalar $\gamma$ such that for all $k$

$$
\left\|e^{k}\right\| \leq \frac{\gamma}{k+1}
$$

Proof. We have, using eqs. (37) and (38),

$$
\begin{aligned}
e^{k}= & \sum_{i=1}^{m}\left(1-\lambda_{k+1}^{m-i}\right) \nabla g_{i}\left(x^{k}\right) g_{i}\left(x^{k}\right) \\
& +\sum_{i=1}^{m} \lambda_{k+1}^{m-i}\left(\nabla g_{i}\left(x^{k}\right) g_{i}\left(x^{k}\right)-\nabla g_{i}\left(\psi_{k m+i-1}\right) g_{i}\left(\psi_{k m+i-1}\right)\right)
\end{aligned}
$$

so from assumptions (32) and (33) we obtain

$$
\begin{aligned}
\left\|e^{k}\right\| & \leq\left(1-\lambda_{k+1}^{m}\right) \sum_{i=1}^{m}\left\|\nabla g_{i}\left(x^{k}\right) g_{i}\left(x^{k}\right)\right\|+L \sum_{i=1}^{m}\left\|x^{k}-\psi_{k m+i-1}\right\| \\
& \leq \frac{c M}{k+1}+L \sum_{i=1}^{m}\left\|x^{k}-\psi_{k m+i-1}\right\|,
\end{aligned}
$$

where $M$ is a bound for $\sum_{i=1}^{m}\left\|\nabla g_{i}\left(x^{k}\right) g_{i}\left(x^{k}\right)\right\|$. We also have, using eq. (21), for all $k$ and $i \geq 2$,

$$
\left\|x^{k}-\psi_{k m+i-1}\right\| \leq\left\|H_{k m}^{-1}\right\| \sum_{j=1}^{i-1}\left\|\nabla g_{i}\left(\psi_{k m+j}\right) g_{i}\left(\psi_{k m+j}\right)\right\| .
$$


Using the boundedness of $\psi_{i}$ and Lemma 2, we see that for all $i$ and some $\delta>0$ we have for $k \geq 1$

$$
\left\|x^{k}-\psi_{k m+i-1}\right\| \leq \frac{\delta}{k} \leq \frac{2 \delta}{k+1} .
$$

Combining this relation with eq. (40), we obtain the desired relation (39).

Assumption (32) together with eq. (37) implies that

$$
\|\nabla f(x)-\nabla f(y)\| \leq m L\|x-y\| \quad \forall x, y \in \Re^{n} .
$$

The next lemma is a well-known consequence of this relation. We include the proof for completeness.

Lemma 4. For all $x$ and $y$, there holds

$$
f(x+y) \leq f(x)+y^{\prime} \nabla f(x)+\frac{m L}{2}\|y\|^{2} .
$$

Proof. Let $t$ be a scalar parameter and let $F(t)=f(x+t y)$. Using eq. (41), we have

$$
\begin{aligned}
f(x+y)-f(x) & =F(1)-F(0)=\int_{0}^{1} \frac{d F}{d t}(t) d t=\int_{0}^{1} y^{\prime} \nabla f(x+t y) d t \\
& \leq \int_{0}^{1} y^{\prime} \nabla f(x) d t+\left|\int_{0}^{1} y^{\prime}(\nabla f(x+t y)-\nabla f(x)) d t\right| \\
& \leq \int_{0}^{1} y^{\prime} \nabla f(x) d t+\int_{0}^{1}\|y\| \cdot\|\nabla f(x+t y)-\nabla f(x)\| d t \\
& \leq y^{\prime} \nabla f(x)+\|y\| \int_{0}^{1} L t\|y\| d t \\
& =y^{\prime} \nabla f(x)+\frac{m L}{2}\|y\|^{2} .
\end{aligned}
$$

We are now ready to prove Proposition 2.

Proof of Proposition 2. We have, using the Kalman filter recursion (13) and the definition (38) of $e^{k}$,

$$
x^{k+1}=x^{k}-H_{(k+1) m}^{-1}\left(\sum_{i=1}^{m} \lambda_{k+1}^{m-i} \nabla g_{i}\left(\psi_{k m+i-1}\right) g_{i}\left(\psi_{k m+i-1}\right)\right)=x^{k}+d^{k},
$$

where

$$
d^{k}=-H_{(k+1) m}^{-1}\left(\nabla f\left(x^{k}\right)-e^{k}\right) .
$$

Using Lemmas 2 and 3 and the fact that

$$
\frac{1}{c_{2}(k+1)} \leq\left\|H_{(k+1) m}^{-1}\right\| \leq \frac{1}{c_{1}(k+1)},
$$

which follows from Lemma 2 , it is seen that

$$
\begin{aligned}
d^{k^{\prime}} \nabla f\left(x^{k}\right) & =-\nabla f\left(x^{k}\right)^{\prime} H_{(k+1) m}^{-1} \nabla f\left(x^{k}\right)+e^{k^{\prime}} H_{(k+1) m}^{-1} \nabla f\left(x^{k}\right) \\
& \leq-\frac{\left\|\nabla f\left(x^{k}\right)\right\|^{2}}{c_{2}(k+1)}+\frac{\left\|e^{k}\right\|\left\|\nabla f\left(x^{k}\right)\right\|}{c_{1}(k+1)} \\
& \leq-\frac{\left\|\nabla f\left(x^{k}\right)\right\|^{2}}{c_{2}(k+1)}+O\left(\frac{1}{(k+1)^{2}}\right)\left\|\nabla f\left(x^{k}\right)\right\|
\end{aligned}
$$


and

(44)

$$
\begin{aligned}
\left\|d^{k}\right\|^{2} & \leq\left\|H_{(k+1) m}^{-1}\right\|^{2}\left(\left\|\nabla f\left(x^{k}\right)\right\|+\left\|e^{k}\right\|\right)^{2} \\
& =O\left(\frac{1}{(k+1)^{2}}\right)\left(\left\|\nabla f\left(x^{k}\right)\right\|+O\left(\frac{1}{k+1}\right)\right)^{2} \\
& =O\left(\frac{1}{(k+1)^{2}}\right)\left\|\nabla f\left(x^{k}\right)\right\|^{2}+O\left(\frac{1}{(k+1)^{3}}\right)\left\|\nabla f\left(x^{k}\right)\right\|+O\left(\frac{1}{(k+1)^{4}}\right) .
\end{aligned}
$$

Using these relations in eq. (42), we obtain

$$
\begin{aligned}
f\left(x^{k+1}\right) \leq f\left(x^{k}\right) & +d^{k^{\prime}} \nabla f\left(x^{k}\right)+\frac{m L}{2}\left\|d^{k}\right\|^{2} \\
\leq f\left(x^{k}\right) & -\left(\frac{1}{c_{2}(k+1)}+O\left(\frac{1}{(k+1)^{2}}\right)\right)\left\|\nabla f\left(x^{k}\right)\right\|^{2} \\
& +O\left(\frac{1}{(k+1)^{2}}\right)\left\|\nabla f\left(x^{k}\right)\right\|+O\left(\frac{1}{(k+1)^{4}}\right) .
\end{aligned}
$$

Thus, since $\left\|\nabla f\left(x^{k}\right)\right\|$ is bounded, there exist constants $\beta_{1}>0$ and $\beta_{2}>0$ and a positive integer $\bar{k}$ such that

$$
f\left(x^{k+1}\right) \leq f\left(x^{k}\right)-\frac{\beta_{1}}{k}\left\|\nabla f\left(x^{k}\right)\right\|^{2}+\frac{\beta_{2}}{k^{2}} \quad \forall k \geq \bar{k}
$$

It is well known that if $\left\{u^{k}\right\}$ and $\left\{d^{k}\right\}$ are nonnegative sequences such that $u^{k+1} \leq$ $u^{k}+\delta^{k}$ for all $k$ and $\sum_{k=1}^{\infty} \delta^{k}<\infty$, then $\left\{u^{k}\right\}$ converges; this is a special case of the supermartingale convergence theorem (see, e.g., [Pol87, p. 49] or [BeT89, p. 677]). Since $f(x) \geq 0$ for all $x$, it follows from eq. (45) that $\left\{f\left(x^{k}\right)\right\}$ converges.

From eq. (45) we have for all $k \geq \bar{k}$

$$
f\left(x^{k+1}\right) \leq f\left(x^{\bar{k}}\right)-\sum_{i=\bar{k}}^{k} \frac{\beta_{1}}{i}\left\|\nabla f\left(x^{i}\right)\right\|^{2}+\sum_{i=\bar{k}}^{k} \frac{\beta_{2}}{i^{2}}
$$

Since $\sum_{i=\bar{k}}^{\infty} 1 / i=\infty$ and $\sum_{i=\bar{k}}^{\infty} 1 / i^{2}<\infty$, we see also that there cannot exist an $\epsilon>0$ such that $\left\|\nabla f\left(x^{k}\right)\right\|^{2}>\epsilon \forall k \geq \bar{k}$. Therefore, we must have $\liminf _{k \rightarrow \infty}\left\|\nabla f\left(x^{k}\right)\right\|=0$.

We will now show that $\left\|\nabla f\left(x^{k}\right)\right\| \rightarrow 0$. Indeed, assume the contrary, that is, there exists an $\epsilon>0$ such that $\left\|\nabla f\left(x^{k}\right)\right\|>\epsilon$ for all $k$ in an infinite subset of integers $\mathcal{K}$. For each $k \in \mathcal{K}$, let $i(k)$ be the first index $i$ such that $i>k$ and $\left\|\nabla f\left(x^{i}\right)\right\|<\epsilon / 2$, so that

$$
\frac{\epsilon}{2} \leq\left\|\nabla f\left(x^{k}\right)\right\|-\left\|\nabla f\left(x^{i(k)}\right)\right\| \leq\left\|\nabla f\left(x^{k}\right)-\nabla f\left(x^{i(k)}\right)\right\| \leq L\left\|x^{k}-x^{i(k)}\right\| \leq L \sum_{i=k}^{i(k)-1}\left\|d^{i}\right\| .
$$

Since from eq. (44) we have $\left\|d^{k}\right\|=O(1 / k)$, eq. (47) implies that for some constant $B_{1}>0$,

$$
\frac{\epsilon}{2} \leq B_{1} \sum_{i=k}^{i(k)-1} \frac{1}{i} \quad \forall k \in \mathcal{K}
$$


From eq. (46) we see that

$$
f\left(x^{i(k)}\right) \leq f\left(x^{k}\right)-\beta_{1}\left(\frac{\epsilon}{2}\right)^{2} \sum_{i=k}^{i(k)-1} \frac{1}{i}+\sum_{i=k}^{i(k)-1} \frac{\beta_{2}}{i^{2}} \quad \forall k \in \mathcal{K}
$$

Since $\left\{f\left(x^{k}\right)\right\}$ converges and $\lim _{k \rightarrow \infty} \sum_{i=k}^{i(k)-1} \beta_{2} / i^{2}=0$, it follows that

$$
\lim _{k \rightarrow \infty, k \in \mathcal{K}} \sum_{i=k}^{i(k)-1} \frac{1}{i}=0
$$

contradicting the earlier conclusion that $\frac{\epsilon}{2} \leq B_{1} \sum_{i=k}^{i(k)-1} 1 / i$ for all $k \in \mathcal{K}$. Therefore, $\left\|\nabla f\left(x^{k}\right)\right\| \rightarrow 0$, and it follows that every limit point of $\left\{x^{k}\right\}$ is a stationary point of $f$.

Note that the proof of Lemma 2 carries through even if the initial matrix $H_{0}$ is any positive-definite matrix rather than $H_{0}=0$. As a result, Proposition 2 also holds when $H_{0}$ is some positive-definite matrix, in which case it is unnecessary to assume that the matrices $\nabla g_{i}(x) \nabla g_{i}(x)^{\prime}$ have full rank, as long as enough alternative assumptions are imposed to guarantee the validity of the crucial Lemma 2 . More generally, our method of proof shows that the convergence characteristics of the method are maintained by any scheme that varies $\lambda$ and/or $H$ in a way that Lemma 2 holds.

The boundedness assumption on the sequence of vectors $\psi_{i}$ is a substantial weakness of Proposition 2. It is not easy to remove this assumption because the algorithm does not have an explicit stepsize mechanism to control the magnitude of the initial iterates. On the other hand, one can employ the device of projecting the iterates $\psi_{i}$ on a compact set that is known to contain an optimal solution and use a projection version of the EKF of the type introduced in [Ber82a] and [Ber82b, §1.5]. Projecting the iterates on a compact set is a well-known approach to enhance the theoretical convergence properties of the EKF (see [Lju79]).

In practice, the method seems to converge considerably faster if $\lambda$ is initially less than 1 and is progressively increased toward 1 in a judicious manner. On the other hand, an implicit diminishing stepsize as indicated by Lemma 2 is essential for the convergence of the method, and such a stepsize induces a generically sublinear convergence rate. This characteristic is shared with the backpropagation method where, to achieve a linear convergence rate, it is essential to use a stepsize that is bounded away from zero, but when such a stepsize is used, the method tends to converge to oscillate [Luo91].

We finally note that as a result of its sublinear convergence rate, the EKF will typically become ultimately slower than the Gauss-Newton method, even though it may be much faster in the initial iterations. The ultimate convergence rate of both the EKF and the backpropagation method may be improved by modifications that gradually change the incremental character of these methods and ultimately make them identical to the pure Gauss-Newton method and the steepest-descent method, respectively. In particular, as convergence is approached, one may adaptively combine ever larger groups of data blocks together into single data blocks. When all data blocks are combined into a single block, the EKF and the backpropagation method will become equivalent to the pure Gauss-Newton method and the steepest-descent method, respectively. 
4. Conclusions. In this paper we have considered EKF algorithms for least squares problems, which consist of repeated cycles through the data set. The computational significance of these algorithms is well known from the control theory literature and has been documented by Davidon [Dav76] in the optimization literature. The algorithms are incremental in nature and bear a similar relation to the GaussNewton method as incremental backpropagation methods bear to the steepest-descent method. Because of their incremental character, EKF methods seem particularly well suited for neural network training problems. However, there has been no convergence analysis of these methods for the case of a finite data set, and the present paper fills this gap.

\section{REFERENCES}

[AWT69] M. Athans, R. P. Wishner, AND A. Bertolini, Suboptimal state estimation for continuous time nonlinear systems from discrete noisy measurements, IEEE Trans. Automat. Control, AC-13 (1968), pp. 504-514.

[AnM79] B. D. O. Anderson And J. B. Moore, Optimal Filtering, Prentice-Hall, Englewood Cliffs, NJ, 1979.

[BeT89] D. P. Bertsekas and J. N. Tsitsiklis, Parallel and Distributed Computation: Numerical Methods, Prentice-Hall, Englewood Cliffs, NJ, 1989.

[BeT96] -, Neuro-Dynamic Programming, Athena Scientific, Belmont, MA, 1996.

[Bel94] B. M. BELL, The iterated Kalman smoother as a Gauss-Newton method, SIAM J. Optim., 4 (1994), p. 626.

[Ber82a] D. P. BeRTSEKAS, Projected Newton methods for optimization problems with simple constraints, SIAM J. Control Optim., 20 (1982), pp. 221-246.

[Ber82b] - Constrained Optimization and Lagrange Multiplier Methods, Academic Press, New York, 1982.

[Ber95] - Nonlinear Programming, Athena Scientific, Belmont, MA, 1995.

[Dav76] W. C. Davidon, New least squares algorithms, J. Optim. Theory Appl., 18 (1976), pp. 187-197.

[Gai93] A. A. Gaivoronski, Convergence Analysis of Parallel Backpropagation Algorithm for Neural Networks, Symposium on Parallel Optimization 3, Madison, WI, July 7-9, 1993.

[Gri93] L. GrIPPo, A Class of Unconstrained Minimization Methods for Neural Network Training, Symposium on Parallel Optimization 3, Madison, WI, July 7-9, 1993.

[Jaz70] A. H. JAzwinski, Stochastic Processes and Filtering Theory, Academic Press, New York, 1970.

[KuC78] H. J. Kushner And D. S. Clark, Stochastic Approximation Methods for Constrained and Unconstrained Systems, Springer-Verlag, New York, 1978.

[Lju77] L. LJUnG, Analysis of recursive stochastic algorithms, IEEE Trans. Automat. Control, AC-22 (1977), pp. 551-575.

[Lju79] - Asymptotic behavior of the extended Kalman filter as a parameter estimator for linear systems, IEEE Trans. on Automat. Control, AC-24 (1979), pp. 36-50.

[LuT93] Z. Q. LUO AND P. TSENG, Analysis of an approximate gradient projection method with applications to the backpropagation algorithm, Optim. Meth. Software, 4 (1994), pp. 85101.

[Luo91] Z. Q. Luo, On the convergence of the LMS algorithm with adaptive learning rate for linear feedforward networks, Neural Comput., 3 (1991), pp. 226-245.

[MaS94] O. L. MANGASARIAN AND M. V. Solodov, Serial and parallel backpropagation convergence via nonmonotone perturbed minimization, Optim. Methods Software, 4 (1994), pp. $103-116$.

[Man93] O. L. MANGASARIAN, Mathematical programming in neural networks, ORSA J. Comput., 5 (1993), pp. 349-360.

[Meh71] R. K. MEHRA, A comparison of several nonlinear filters for reentry vehicle tracking, IEEE Trans. Automat. Control, AC-16 (1971), pp. 307-319.

[Pap82] T. N. PAPPAS, Solution of Nonlinear Equations by Davidon's Least Squares Method, M.S. thesis, Dept. of Electrical Engineering and Computer Science, Massachusetts Institute of Technology, Cambridge, MA, 1982. 
[PoT73] B. T. PolJAK AND Y. Z. TsYPKIN, Pseudogradient adaptation and training algorithms, Automation Remote Control, 12 (1973), pp. 45-68.

[Pol87] B. T. POLJAK, Introduction to Optimization, Optimization Software Inc., NY, 1987.

[RRK92] D. W. RuCK, S. K. Rogers, M. KABrisky, P. S. MAYBeCK, AND M. E. Oxley, Comparative analysis of backpropagation and the extended Kalman filter for training multilayer perceptrons, IEEE Trans. Pattern Analysis and Machine Intelligence, 14 (1992), pp. 686691.

[TBA86] J. N. Tsitsiklis, D. P. Bertsekas, and M. Athans, Distributed asynchronous deterministic and stochastic gradient optimization algorithms, IEEE Trans. Automat. Control, AC-31 (1986), pp. 803-812.

[THS77] R. R. TENney, R. S. HebBert, AND N. R. SANDEll, JR., Tracking filter for maneuvering sources, IEEE Trans. Automat. Control, AC-22 (1977), pp. 246-251.

[Tsi84] J. N. Tsitsiklis, Problems in Decentralized Decision Making and Computation, Ph.D. dissertation, Dept. of Electrical Enginering and Computer Science, Massachusetts Institute of Technology, Cambridge, MA, 1984.

[Urs80] B. URsin, Asymptotic convergence properties of the extended Kalman filter using filtered state estimates, IEEE Trans. Automat. Control, AC-25 (1980), pp. 1207-1211.

[Whi89a] H. WHITE, Some asymptotic results for learning in single hidden-layer feedforward network models, J. Amer. Statist. Assoc., 84 (1989), pp. 1003-1013.

[Whi89b] - Learning in artificial neural networks: A statistical perspective, Neural Comput., 1 (1989), pp. 425-464.

[WaT90] K. WATANABE AND S. G. TzAFESTAS, Learning algorithms for neural networks with the Kalman filters, J. Intelligent Robotic Systems, 3 (1990), pp. 305-319.

[WeM80] H. WEISS AND J. B. MOORE, Improved extended Kalman filter design for passive tracking, IEEE Trans. Automat. Control, AC-25 (1980), pp. 807-811.

[WiH60] B. Widrow AND M. E. HoFF, Adaptive Switching Circuits, Institute of Radio Engineers, Western Electronic Show and Convention, Convention Record, part 4, 1960, pp. 96-104.

[WiS85] B. Widrow And S. D. Stearns, Adaptive Signal Processing, Prentice-Hall, Englewood Cliffs, NJ, 1985. 